\title{
Evolutions of Neutron Stars and their Magnetic Fields
}

\section{G. S. Bisnovatyi-Kogan*}

Space Research Institute, Russian Academy of Sciences, Profsoyuznaya 84/32, Moscow 117997, Russia

and

National Research Nuclear University MEPhI

(Moscow Engineering Physics Institute), Kashirskoe Shosse 31, Moscow 115409, Russia

E-mail: gkoganeiki.rssi.ru

Estimations of magnetic fields of neutron stars, observed as radio and X-ray pulsars, are discussed. It is shown, that theoretical and observational values for different types of radio pulsars are in good correspondence. Radio pulsars in close binaries and millisecond pulsars, which have passed the stage of disk accretion (recycled radio pulsars), have magnetic fields 2-4 orders of magnitude smaller than ordinary single pulsars. Most probably, the magnetic field of the neutron star was screened by the infalling material. Several screening models are considered. Formation of single recycled pulsars loosing its companion is discussed. Magnetic fields of some X-ray pulsars are estimated from the cyclotron line energy. In the case of Her X-1 this estimation exceeds considerably the value of its magnetic field obtained from long term observational data related to the beam structure evolution. Another interpretation of the cyclotron feature, based on the relativistic dipole radiation mechanism, could remove this discrepancy. Observational data about soft gamma repeaters and their interpretation as magnetars are critically analyzed.

XII Multifrequency Behaviour of High Energy Cosmic Sources Workshop

12-17 June 2017

Mondello (Palermo), Italy

\footnotetext{
*Speaker.
} 


\section{Introduction}

First theoretical estimations of neutron star magnetic fields have been obtained from the condition of the magnetic flux conservation during contraction of a normal star to a neutron star [36]:

$$
B_{n s}=B_{s}\left(\frac{R_{s}}{R_{n s}}\right)^{2} \text {. }
$$

For main sequence stellar magnetic field $B_{s}=10 \div 100 \mathrm{G}$, and stellar radius $R_{s}=(3 \div 10) R_{\odot} \approx$ $(2 \div 7) 10^{11} \mathrm{~cm}$, we get $B_{n s} \approx 4 \times 10^{11} \div 5 \times 10^{13} \mathrm{G}$, for a neutron star radius $R_{n s}=10^{6} \mathrm{~cm}$. As shown below, this simple estimation occurs to be in a good correspondence with most observational data.

Neutron stars are born with magnetic fields comparable with the fields of youngest pulsars. The youngest and the best studied Crab pulsar PSR 0531+21 is about 1000 years old and has a magnetic field about $3.5 \cdot 10^{12} \mathrm{G}$. The pulsar in Vela PSR 0833-45 is 10 times older, but has almost the same magnetic field. Magnetic fields of some oldest pulsars PSR 0826-34 $\left(3 \cdot 10^{7}\right.$ years old $)$ and PSR $1819-22\left(3 \cdot 10^{7}\right.$ years old $)$ are equal to $1.4 \cdot 10^{12}$ and $1.1 \cdot 10^{12} \mathrm{G}$ respectively [57]. The simple comparison of these data gives the indication to low, or may be negligible, damping of the magnetic field due to Ohmic losses inside the neutron star matter. This conclusion seems to be plausible, because in very dense strongly degenerate layers of the neutron star, where the electrical current, producing magnetic fields could flow, the electrical conductivity is enormously high, so in this case we may expect practically no Ohmic damping. These simple estimations had been confirmed by the detailed statistical analysis of the big sample of single radio pulsars [5], where the conclusion about low (or negligible) Ohmic damping of magnetic fields of radio pulsars was obtained quite reliably. Magnetic fields of different types of neutron stars are reviewed in [13, 15].

\section{Radio pulsars}

It is now commonly accepted that radio pulsars are rotating neutron stars with inclined magnetic axes. For rotating with angular velocity $\Omega$ dipole, the rotational energy losses $[48,73]$ are determined as $\dot{E}=A B^{2} \Omega^{4}$. The rotational energy is $E=\frac{1}{2} I \Omega^{2}, I$ is an inertia momentum of the neutron star. By measuring of $P=2 \pi / \Omega$ and $\dot{P}$, we obtain the observational estimation of the neutron star magnetic field as

$$
B^{2}=\frac{I P \dot{P}}{4 \pi^{2} A} .
$$

It was shown by [38] that energy losses by a pulsar relativistic wind are important also when the magnetic and rotational axes coincide, and one may use (2.1) with $A=\frac{R_{n s}^{6}}{6 c^{3}}$, and $B_{n s}$ corresponding to the magnetic pole, at any inclination angle. Magnetic fields of radio pulsars estimated using (2.1) with observational values of $P$ and $\dot{P}$ lay in a wide region between $10^{8}$ and $10^{13} \mathrm{G}$ [55]. There are two distinctly different groups: single radio pulsars with periods exceeding $0.033 \mathrm{sec}$, and magnetic fields between $10^{11}$ and $10^{13} \mathrm{G}$, and recycled pulsars (RP), present or former members of close binary systems with millisecond periods and low magnetic fields between $10^{8}$ and $10^{10}$ G. Low magnetic field of recycled pulsars is probably a result of its damping during preceding accretion stage [20,22]. 


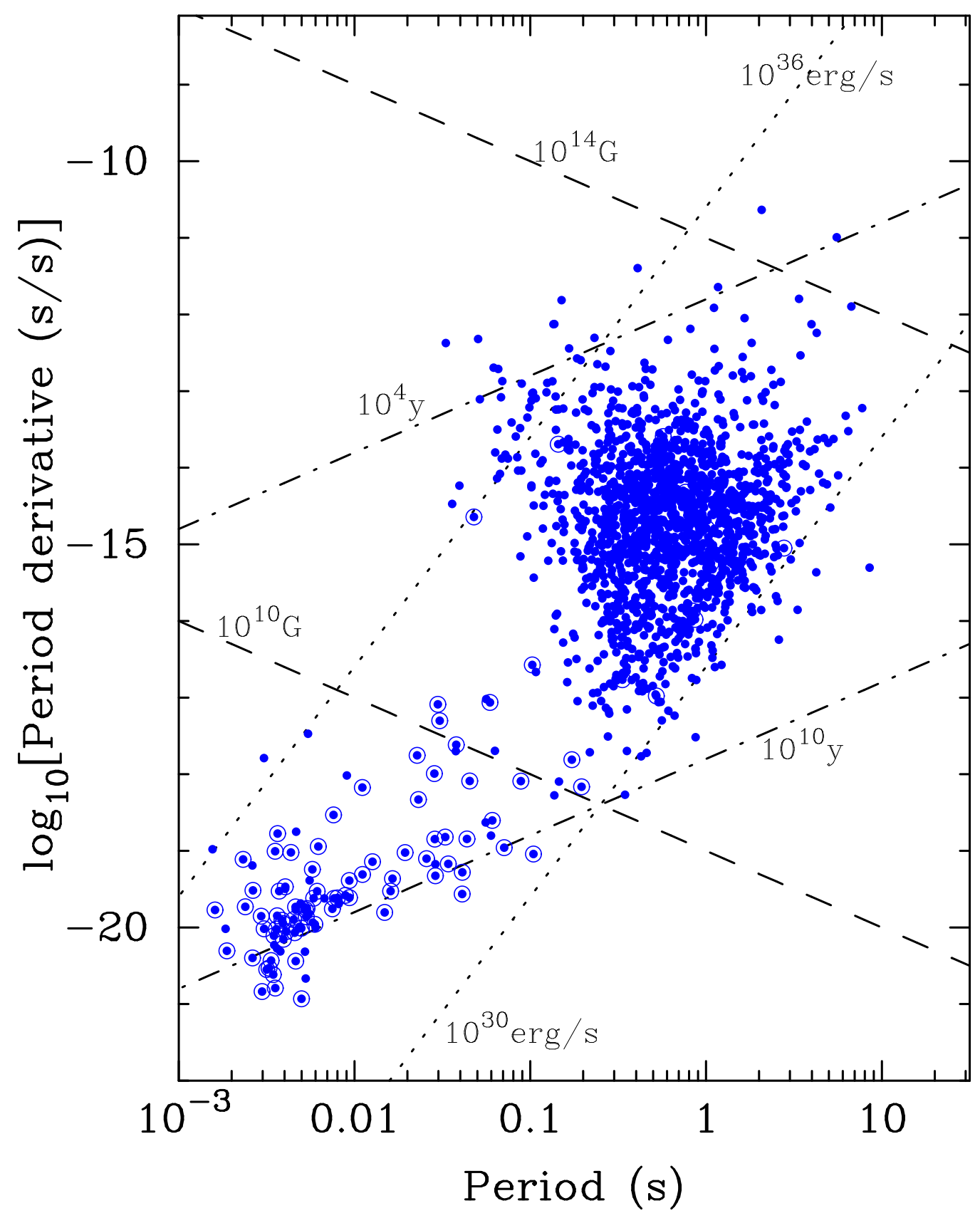

Figure 1: $P$ - $\dot{P}$ diagram showing the current sample of radio pulsars. Binary pulsars are highlighted by open circles. Lines of constant magnetic field (dashed), characteristic age (dash-dotted) and spin-down energy loss rate (dotted) are also shown. from [51]. 
The pulsars with a small magnetic field in the left lower angle decrease their magnetic field during recycling by accretion in a close binary. see [20,14].

Soft Gamma Repeaters (SGR) are single neutron stars with rotational periods $2 \div 8$ seconds They produce "gamma-ray giant bursts", when their luminosity $L$ in the peak increase $5 \div 6$ orders of magnitude. Having a slow rotation, and small rotational energy, their observed average luminosity exceeds rotational loss of energy more than 10 times, and orders of magnitude during the giant outbursts. It was suggested by in [32], that the source of energy is their huge magnetic field, 2 or 3 order of a magnitude larger, then the average field in radio pulsars. Such objects were called magnetars $[31,32]$.

\section{Recycled radio pulsars}

During several years after discovery of pulsars only single objects had been found. It was an impression, that pulsars avoid binaries. When half (or even more) stars are binaries, this phenomena was explained either by pair disruption during supernova explosion leading to pulsar formation, or by absence of SN explosions at the end of evolution of stars in close binaries [87].

Detailed analysis of the fate of close X-ray binaries in low-mass systems, namely Her X-1, was done in [20], and the conclusion was made that evolution of such system will be ended by formation of a non-accreting neutron star in close binary, which should become a radio pulsar. It was shown, that neutron star rotation is accelerated during disk accretion stage, so the second time born (recycled) radio pulsar should become visible, provided it has a magnetic field similar to other single pulsars. Absence of radio pulsars in close binaries, in spite of intensive searches could be explained by the only reason: during accretion stage the magnetic field of the neutron star is screened by the inflowing accreting gas, so the recycled pulsar should have $B \sim 10^{8}-10^{10}$ G, 2-4 orders of magnitude smaller than average field strength of radio pulsars. Discovery of the first binary pulsar [41], and subsequent discovery of more than 100 recycled pulsars [51, 56] had confirmed this conclusion: all recycled pulsars have small values of magnetic fields, as was predicted in [20].

A simple estimation of the magnetic field screening during accretion had shown a large potential possibility of such process. It was estimated in [10] that in absence of instabilities leading to penetration of the infalling plasma into pulsar magnetosphere, the pressure of the accreting gas exceeds the magnetic pressure of the dipole with $B=10^{12} \mathrm{G}$. already after one day of accretion at subcritical accretion rate $10^{-9} M_{\odot} /$ year, and the original magnetic dipole is completely graved under a plasma layer, and currents in the plasma prevent external appearance of stellar magnetic field. In reality the instability and gas penetration through the magnetosphere make the screening process much slower, and when the penetration layer reaches the surface of the star the external magnetic field strength of the neutron star could reach the stationary state.

From the statistical analysis of 24 binary radio pulsars with nearly circular orbits and low mass companions, it was discovered [90] a clear correlation between spin period $P_{p}$ and orbital period $P_{o r b}$, as well as between the magnetic field and orbital period: pulsar period and magnetic field strength increases with the orbital period at $P_{\text {orb }}>100$ days, and scatters around $P_{p} \sim 3 \mathrm{~ms}$ and $B \sim$ $2 \cdot 10^{8} \mathrm{G}$ for smaller binary periods. These relations strongly suggest that an increase in the amount of accreted mass leads to a decay of the magnetic field, and a 'bottom' field strength of about $10^{8} \mathrm{G}$ 
is also implied. Several models of magnetic field screening during accretion had been considered, see $[27,28]$ and references therein. Evolutionary aspects of formation of recycled pulsars are discussed in [20,89], and summarized in [4]. Several scenario had been analyzed in [20,89] for the evolution of binaries, massive enough to produce the neutron star or black hole in one or both remnants. During the evolution such stars go through stages of different nuclear burning, which are ended by formation of carbon-oxygen white dwarf, or proceeds until formation of the iron peak elements core, which collapses with formation of the neutron star or a black hole. The first born relativistic star (neutron, or black hole) goes through the stage of an accreting binary X-ray source, and the evolution of such system is finished after end of evolution of the second component, and the end of accretion. During the evolution of massive close binary there are possibilities of a disruption of the binary after the first or the second collapse, which may be accompanied by explosion with large mass ejection, or by a kick due to anisotropy in a mass ejection or in neutrino flux. It was nevertheless concluded in [20], that "evolutionary analysis cannot exclude formation of close binary, containing neutron star, and another star on the last evolutionary state (white dwarf, neutron star, black hole). Contrary, this analysis indicates to formation of such binaries at the end of evolution of sufficiently massive systems with a large probability." Same conclusion was obtained in [89]. The absence of discovery of binary pulsars in 1973 was unambiguously explained in [20] as follows: "The absence of radio pulsars in close binaries may be explained if we suggest additionally, that magnetic field of the neutron star is damped during accretion stage"

The probabilistic conclusion about formation of radio pulsars in close binaries was definitely confirmed in [20] by the analysis of the evolution of one of the best learned X-ray pulsars Her $\mathrm{X}$-1. The component of the neutron star in this system is a low mass star which will end its life by formation of the white dwarf, when the pair cannot be disrupted. On the other hand, during accretion the neutron star acquires a rapid rotation, and it was shown in [20], that during the process of termination of accretion the neutron star - X-ray pulsar cannot follow the stationary period, increasing with decreasing of the the accretion rate $\dot{M}$, and after the end of accretion will remain to be rather rapidly rotating neutron star with a period of the order, or smaller than periods of the known radio pulsars. So, the only explanation of absence of binary radio pulsars in to 1973 year remains their faintness, connected with low magnetic field.

The first proof of this conclusion came soon by discovery of the first binary radio pulsar PSR $1913+16$ [41]. This pulsar was a rapidly rotating with a period $0.059 \mathrm{~s}$ in the close binary with a period $7^{h} 45^{m}$. Just after the discovery I have identified this object with the old recycled pulsar with low magnetic field [9]. Measurements [86] of the period derivative $\dot{P}$, which gave possibility to estimate the age of this pulsar $\tau=\frac{P}{2 P} \approx 10^{8}$ years, and magnetic field $B \approx 2 \cdot 10^{10} \mathrm{G}$ had proofed this identification. Among more than 100 recycled pulsars most objects consist of a neutron star with low mass white dwarf companion, and the systems with two neutron stars, like in PSR 1913+16 are in minority. Double-neutron-star (DNS) binaries are rare, only $\sim 10$ such systems are known. All recycled pulsars, with white dwarf or neutron star companions, have magnetic fields $10^{8}-10^{10}$, so the hypothesis about field damping on the accretion stage seems to be correct.

Another convincing evidence in favor of magnetic field graving during accretion appeared by discovery of the first binary system, containing two radio pulsars. The radio pulsar J0737-3039A with 23-ms period was discovered, which was found to be in a 2.4-hr eccentric orbit with another compact object that the observed orbital parameters suggested was another neutron star [26]. The 
2.8-sec pulsar J0737-3039B was discovered [56] as the companion to the pulsar J0737-3039A in a highly-relativistic double-neutron-star system, allowing unprecedented tests of fundamental gravitational physics. The short orbital period and compactness of the system, and the high timing precision made possible by the large flux density and narrow pulse features of this pulsar promise to make this system a superb laboratory for the investigation of relativistic astrophysics. The resulting in-spiral will end in coalescence of the two stars in about $85 \mathrm{My}$. This discovery significantly increases the estimates of the detection rate of DNS in-spirals by gravitational wave detectors [26]. The clock-like properties of pulsars moving in the gravitational fields of their unseen neutron-star companions have allowed unique tests of general relativity and provided evidence for gravitational radiation.

The properties of these pulsars fit very well the evolutionary scheme of formation of binary pulsars from [20]. According to this scheme the $23 \mathrm{~ms}$ pulsar had accelerated its rotation during accretion, when its magnetic field was decreased due to screening of the accreting material. The second $2.8 \mathrm{~s}$ pulsar was born later in SN explosion, which did not disrupt the pair. Therefore, $\mathrm{ms}$ pulsar should be older, and have weaker magnetic field. Indeed, $23 \mathrm{~ms}$ pulsar has $B=6.3 \times 10^{9}$ $\mathrm{G}$, and characteristic age $\tau=210 \mathrm{My}$; and $2.8 \mathrm{~s}$ pulsar has $B=1.6 \times 10^{12} \mathrm{G}$, and $\tau=50 \mathrm{My}$ [56]. The masses of neutron stars in this binary are estimated as $1.34 \mathrm{M}_{\odot}$ for $23 \mathrm{~ms}$, and $1.25 \mathrm{M}_{\odot}$ for 2.8 s pulsars.

\subsection{Magnetic field damping during accretion}

Accretion induced Ohmic heating and dissipation have been investigated in many works (see i.g. references in [29]). This mechanism may work only if the magnetic field is produced by electrical current in the outer layers of the accreting neutron star, which may be heated during accretion. Evidently, the highly degenerate neutron star core is not sensible to this heating and will preserve its very high conductivity. Therefore magnetic field, originated by deep inside electrical currents may become weaker only due to screening by the infalling plasma during accretion [20]. Different models of magnetic field screening during accretion have been considered. It is usually accepted that matter is channelized by the dipole magnetic field and flows to the magnetic poles of the neutron star [19, 2]. As the magnetic field of the neutron star decreases because of the screening due to accreting material, it is less able to channelize the accretion flow and thereby the polar cap widens. One can easily find out how the angular width $\theta_{\mathrm{P}}$ of the polar cap depends on the surface magnetic field $B_{\mathrm{s}}$ of the neutron star (see, for example, [81]). The field line starting from $\theta_{\mathrm{P}}$ at the surface of the neutron star, with a radius $r_{\mathrm{s}}$, is the last closed field line of the dipolar field and passes through the Alfvén radius $r_{\mathrm{A}}$. It easily follows that

$$
\sin \theta_{\mathrm{P}}=\left(\frac{r_{\mathrm{s}}}{r_{\mathrm{A}}}\right)^{1 / 2}
$$

Assuming that the ram pressure of the freely in-falling accreting material at the Alfvén radius equals the magnetic pressure, a few steps of easy algebra give, see e.g. [50]

$$
r_{\mathrm{A}}=(2 G M)^{-1 / 7} r_{\mathrm{s}}^{12 / 7} B_{\mathrm{s}}^{4 / 7} \dot{M}^{-2 / 7},
$$

where $M$ is the mass of the neutron star and $\dot{M}$ the accretion rate. It follows from (3.1) and (3.2) that 


$$
\sin \theta_{\mathrm{P}} \propto B_{\mathrm{S}}^{-2 / 7} .
$$

This is how the polar cap widens with the weakening magnetic field until $\theta_{\mathrm{P}}$ becomes equal to $90^{\circ}$ when (3.3) obviously ceases to hold. On taking $M=10^{33} \mathrm{gm}, \dot{M}=10^{-8} M_{\odot} \mathrm{yr}^{-1}, r_{\mathrm{s}}=10 \mathrm{~km}$, $B_{\mathrm{S}}=10^{12} \mathrm{G}$, we find from (3.2) that $r_{\mathrm{A}} \approx 300 \mathrm{~km}$. Substituting this in (3.1), we conclude that the initial polar cap angle is of order $10^{\circ}$.

The accreting materials falling through the two polar caps flow horizontally towards the equator in both the hemispheres. At the equator, the opposing materials flowing in from the two poles meet, sink underneath the surface (inducing a counter-flow underneath the equator-ward flow at the surface) and eventually settle radially on the neutron star core. With a suitably specified flow having these characteristics, it was studied kinematically in $[45,30]$ how the magnetic field evolves with time, taking into account the fact that the polar cap width changes with the evolution of the magnetic field, thereby altering the velocity field also. It was found in the simulations [30] that the equator-ward flow near the surface is quite efficient in burying the magnetic field underneath the surface. However, when the polar cap opens to $90^{\circ}$, the accretion becomes spherical and radial. It is found that such accretion is not efficient in burying the magnetic field any further. The magnetic field at the surface of the neutron star keeps decreasing until the polar cap opens to $90^{\circ}$, after which the magnetic field is essentially frozen, since the radial accretion cannot screen it any further. If $\theta_{\mathrm{P}, \mathrm{i}}$ is the initial polar cap width, then it follows from (3.3) that the magnetic field would decrease by a factor $\left(\sin 90^{\circ} / \sin \theta_{\mathrm{P}, \mathrm{i}}\right)^{7 / 2}$ from its initial value before it is frozen to an asymptotic value. On taking $\theta_{\mathrm{P}, \mathrm{i}}$ in the range $5^{\circ}-10^{\circ}$, this factor turns out to be about $10^{3}-10^{4}$, exactly the factor by which the magnetic fields of millisecond pulsars are weaker compared to the magnetic fields of ordinary pulsars.

Put another way, the magnetic field freezes when the Alfvén radius becomes equal to the neutron star radius. The asymptotic value of the surface magnetic field can be found directly from (3.2) by setting $r_{\mathrm{A}}$ equal to $r_{\mathrm{s}}$, which gives

$$
B_{\text {asymp }}=(2 G M)^{1 / 4} \dot{M}^{1 / 2} r_{\mathrm{s}}^{-5 / 4} .
$$

On using the various standard values mentioned before, we find $B_{\text {asymp }} \approx 10^{8} \mathrm{G}$. When the magnetic field falls to this value, it can no longer channelize the accretion flow, resulting in the flow becoming isotropic. Such a flow is unable to screen the magnetic field any further. After the accretion phase is over, the neutron star appears as a millisecond pulsar with this magnetic field. This could be the reason why millisecond pulsars are found with magnetic fields of order $10^{8} \mathrm{G}$, see e.g. [54]. A schematic picture of the plasma flow, magnetic field structure, and electrical currents is given in Figs. 2,3.

Note that the accretion flow in the polar region of a magnetized neutron star is sensitive to various magneto-hydrodynamic instabilities and it is difficult to make an assessment of the effectiveness of screening without a full three-dimensional computation which is yet to be attempted.

A simple analytical model of the magnetic field screening in incompressible fluid approximation was considered in $[27,28]$. It was found a very rapid decrease of the magnetic field in the polar cap during a time scale

$\tau_{p} \approx 10^{5} \dot{M}\left(m_{B} / 10^{-3} M_{\odot}\right)$ years, where $m_{B}=\left(B_{\text {asymp }} / B_{0}\right)^{4 / 7} M_{c r}$ with $B_{\text {asymp }}$ from (3.4), initial 


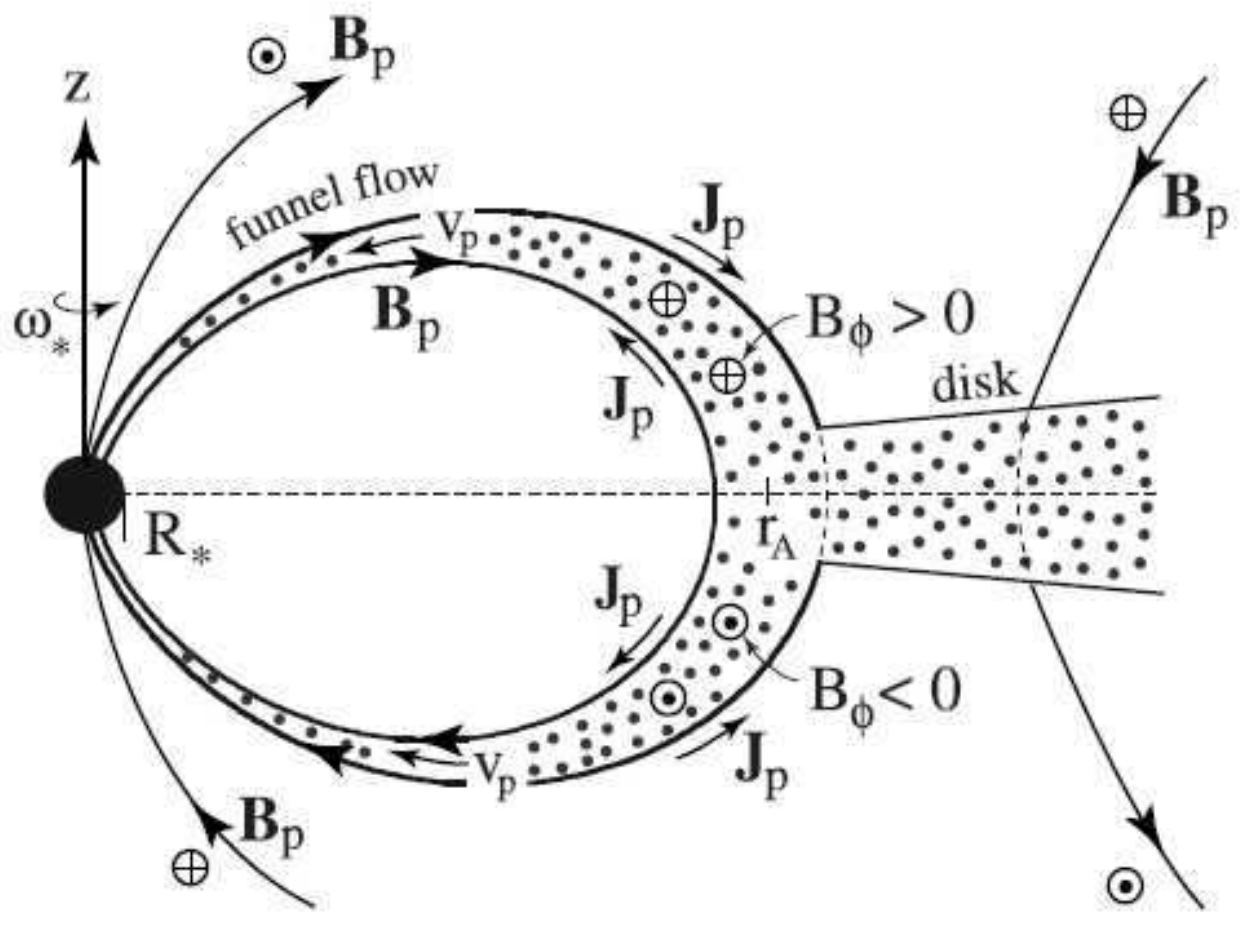

Figure 2: Diagram of the poloidal cross-section of the accretion disk and funnel flow. $\mathrm{B}_{p}$ is the poloidal magnetic field, $\mathrm{B}_{\phi}$ is the toroidal magnetic field, $\mathrm{J}_{p}$ is the poloidal current density, and $\mathrm{v}_{p}$ is the poloidal component of the funnel flow velocity. The dotted circle represents an arrow out of the page, while the circle with the plus sign represents the opposite direction. The solid dots indicate a high plasma density, from [54].

magnetic field of the neutron star $B_{0}$, and mass of the crust $M_{c r}=0.03 M_{\text {odot }}$. Here the polar magnetic field is tending to the same asymptotic value (3.4), and development of instabilities should decrease also the equatorial magnetic field to similar values.

It was noted in [22], that "magnetic fields buried during intensive accretion may start to "percolate" outside after the end of accretion", what could increase the magnetic field of recycled pulsars with time, see also [69]. Some estimations of the field percolation outside have been done in [72]. The authors considered cases with very rapid field increase due to percolation during $10^{3}--10^{4}$ years, up to the initial value which the neutron star had before the start of accretion. Evidently, this conclusion is in contradiction with observation, because all recycled pulsars which could be very old, even up to $10^{10}$ years [51], have equally small magnetic fields. This contradiction is connected with the artificial model considered in [72], where the percolation was started from the depth not more than $260 \mathrm{~m}$, corresponding to the accreted material less that $5 \cdot 10^{-5} M_{\odot}$. The conductivity in the outer crust of the neutron star could be small enough to permit such a rapid percolation. Actually, during the accretion phase lasting $10^{7}-10^{8}$ years the amount of the accreted material is much larger, the magnetic field is buried to much deeper layers with much higher conductivity. Therefore 


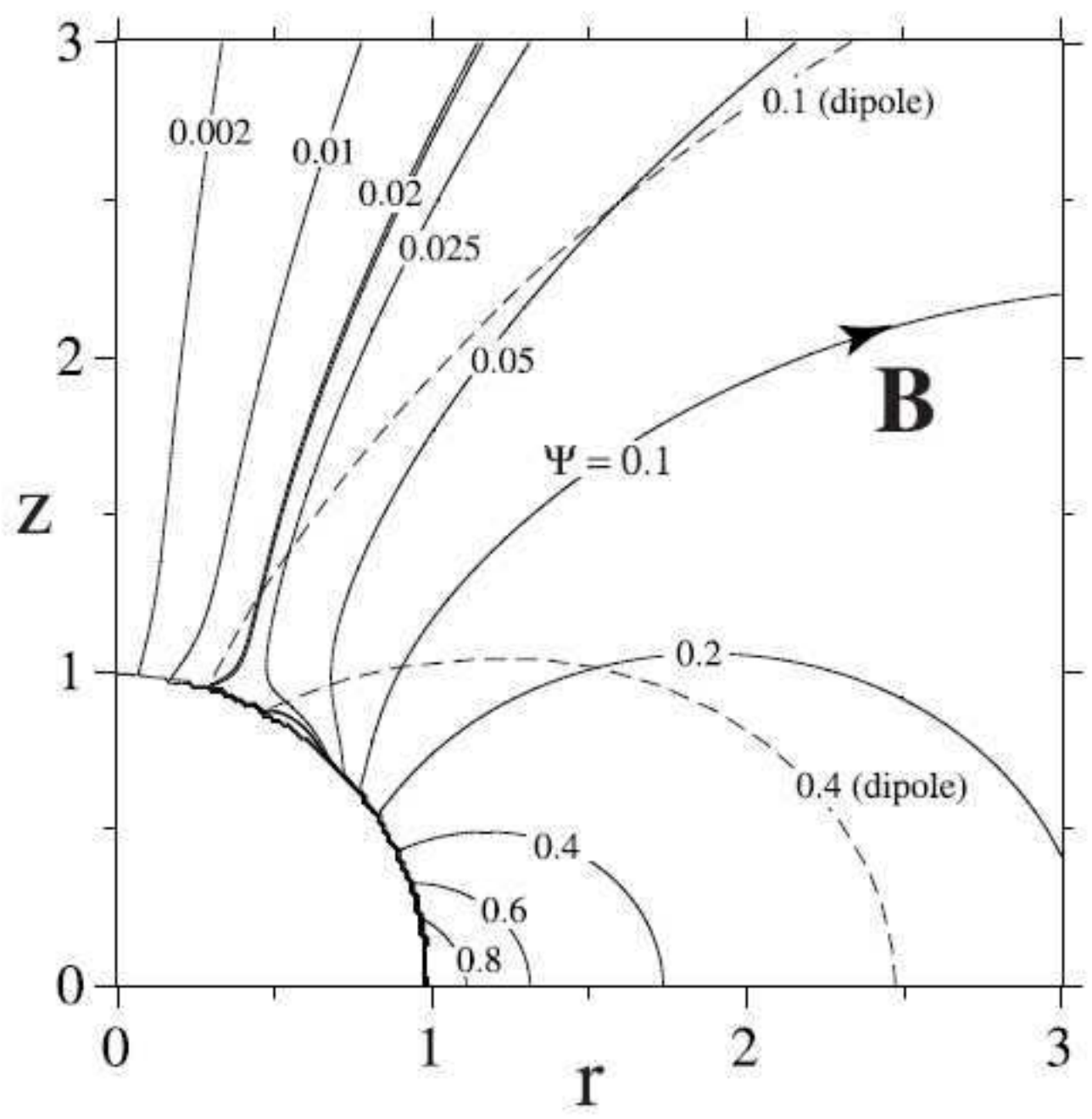

Figure 3: Magnetic field lines of a screened star (solid lines) with three modes at $t / t_{0}=0.85$. The dashed lines are for the initial, pure dipole field. The thicker line is the centroid of the funnel flow. It is clear from this figure that the field of the star is markedly changed by the accretion. Here $t_{0}$ is the time of the field decrease to the stationary state, which is of the order of $10^{5}$ years, from [54].

the outward percolation of the magnetic field in the recycled pulsars should be negligible.

\section{Neutron star evolution in close binaries}

During accretion stage, the neutron star in the close binary is visible as X-ray source, namely, X-ray pulsar. At the end of the companion evolution in HMXB (High mass X-ray Binary) we expect formation of NS+NS binary, or binary with NS+BH (not observed yet), or the binary could be disrupted at the explosion of the companion star. 
In LMXB (Low Mass X-ray Binary) the accretion stage is started also as X-ray source âĂŞ $\mathrm{X}$-ray pulsar, but the evolution of the low-mass star is very slow, so recycling, and accretioninduced decrease of NS magnetic field to its asymptotic value $B_{\text {asymp }} \approx 10^{8} \mathrm{G}$ happens first, and NS becomes as X-ray bursting source, where the recycled pulsar is visible continuously, or only during the outburst $[91,92]$. At the end of evolution of LMXB a rapidly rotating neutron star appears with low magnetic field $\approx 10^{8} \mathrm{G}$. Concentration of LMXB in Globular clusters indicate to their formation in tidal captures, or in triple encounters [23].

\subsection{Formation of single recycled pulsars. Enhanced Evaporation}

The most rapidly rotating stars (ms pulsars) are originated at the end of LMXB evolution. Considerable part of ms recycled pulsars are single, so the question arises: how NS loose its lowmass companion? One of the possibility is an evaporation of low-mass companion by pulsar beam what was suggested after discovery of eclipsing recycled radio pulsar [35, 44], but statistical properties did not confirm it. The concentration of the recycled pulsars (RP) to the globular clusters (GC), about half of RP from the total number $\sim 200$ are situated in GC [51], is very similar to the distribution of low mass X-ray binaries (LMXB). Taking into account, that presently the total relative mass of GC in Galaxy is about $10^{-3}$ and about one half of LMXB are situated in GC, the relative concentration of LBXB in GC is 1000 larger than in the Galaxy [83]. The similarity of distributions of LMXB and RP may be considered as another evidence to their genetic relation [20, 22, 78]. According to [51] there are $\sim 45 \%$ of single RP in GC , and only $\sim 16 \%$ of single RP in the galactic bulge. This evident statistical difference indicates that not only birth of LMXB, but also the disruption of the binaries are connected with close stellar encounters, which are much more frequent in GC than in the bulge [11]. It was suggested in [11], that single RP in the bulge could be the remnants of the completely evaporated GC, same origin may have also LMXB of the bulge [23]. Numerical simulations made in [76] have shown that only pairs with large orbital periods 10 - 100 days could be destroyed by close stellar encounters in globular clusters. However, it follows from observations [51], that RP are usually members of more close binaries. The solution of this problem may be done by the mechanism of "Enhanced Evaporation" (EE), suggested in [12].

It was shown theoretically in [39] and confirmed later by numerical experiments [1], that stellar encounters of stars with close binaries lead to energy extraction from the binary and heating of the cluster field stars. Contrary to that, collisions with sufficiently wide binary lead to its father widening, and finally to its destruction. This property is interpreted very easily. The kinetic energy exchange between stars during encounter is determined by their velocities, almost independently on their single or binary origin. Relaxation processes in stellar encounters lead to establishing of equipartition of energies of all stars. It means, that star with larger kinetic energy lose it, and the one with smaller energy gain it. For single stars it would lead to real equipartition, so that stars with larger energies in average loose it, but another result takes place if one of the encountering objects is a close binary. In close binary star is rapidly rotating on the orbit. If this rotational energy exceeds the average kinetic energy of stars in the cluster, there is an average energy transfer to the field stars. When the star in the binary loose its kinetic energy, its orbit is changing in such a way that is starts to rotate faster than before, and the pair becomes tighter. The binary has an effective negative heat capacity, in accordance with the virial theorem [48] in the binary system. The net loose of the energy by the pair leads to acceleration of the orbital motion. When tight binaries are 
formed in the GC, they could destroy it during time less than the cosmological one, so the LMXB and single RP in the bulge could be formed in such a way.

The situation is quite different, when one of the degenerate members in the close binary fills its Roche Lobe. Let isolated binary contain a neutron star with a mass $M_{1}$, and a degenerate dwarf with a mass $M_{d}$. When the dwarf fills its Roche lobe, the radius of the dwarf $R_{d}$ is connected with a distance between stellar mass centers $R_{12}$ by a relation [74]

$$
R_{d}=R_{12} \frac{2}{3^{4 / 3}}\left(\frac{M_{d}}{M}\right)^{1 / 3}, \quad M=M_{1}+M_{d}
$$

For white dwarfs with an equation of state valid in small mass dwarfs the equilibrium models had been constructed in [93]. The radius of a degenerate dwarf increases with decreasing of its mass until very low masses, when repulsion effects may change this dependence. Gravitation radiation leads to loss of the angular momentum of the system, and tries to make the binary closer, but approach of the dwarf to the neutron star induces a mass transfer. If we suggest that the dwarf fills its Roche lobe, which radius coincides with the dwarf radius, than due to mass transfer dwarf mass decreases, its radius increases with the Roche lobe radius, and the binary is becoming softer. Evolution of such binary under the action of a gravitational radiation was calculated in [12]. It was obtained that during a cosmological time $\tau_{c}=2 \cdot 10^{10}$ years the mass of the carbon dwarf reaches $0.0025 M_{\odot}$. At that time the period of the binary $P$ reaches about 1.5 hours,

$$
P=\frac{2 \pi R_{12}^{3 / 2}}{(G M)^{1 / 2}}=\frac{9 \pi R_{d}^{3 / 2}}{\sqrt{2 G M_{d}}}
$$

and the dwarf velocity in the binary

$$
v_{d}=\frac{2 \pi R_{12}}{P}=\frac{\sqrt{2}}{3^{2 / 3}}\left(\frac{G M^{2 / 3} M_{d}^{1 / 3}}{R_{d}}\right)^{1 / 2}, \quad \text { at } M_{d} \ll M .
$$

The pair becomes soft, and would be destroyed by close encounters with the field stars when the kinetic energy of the dwarf in the binary is less than the average kinetic energy of the field stars: $M_{f} v_{f}^{2}>M_{d} v_{d}^{2}$. For globular clusters 47 Tuc and $\omega$ Cen the corresponding velocities are 10.3 and $16.8 \mathrm{~km} / \mathrm{s}$, and masses are equal to $0.67 M_{\odot}$ and $0.51 M_{\odot}$ respectively. According to calculations [12], the pair in these GC is becoming soft, when the dwarf mass becomes less than $6 \cdot 10^{-4}$ and $9 \cdot 10^{-4}$ respectively. These masses are several times less than the dwarf mass after cosmological time, and to reach these masses one needs about 10 cosmological times.

It was shown in [12], that stellar encounters of the field stars with "hard" pairs, when the binary loose its energy and momentum, act similar to the gravitational radiation. They make the hard pair even harder, but when the dwarf in the pair fills its Roche lobe such encounters make the pair softer. When the encounters with the field stars are effective enough they may transform the hard binary in the state of the mass exchange into the soft one, which would be destroyed directly by close encounters. This process was called in [12] as "enhanced evaporation". For the estimation of the momentum relaxation time $\tau_{m}$ we use the expression [84], which is valid for stars with comparable masses, because in the close binary the centrum mass motion may take the extra momentum 


$$
\tau_{m}=\frac{v_{d}^{3}}{4 \pi G^{2} M_{f}^{2} n_{f} \Lambda} .
$$

Here Coulomb logarithm $\Lambda \approx 10, n_{f}$ is the average density of the field stars. With account of (4.3) the characteristic encounter time, which determines the time of the pair destruction due to EE is written as

$$
\tau_{m} \approx 10^{22} \frac{m m_{d}}{m_{f}^{2} n_{51}} \mathrm{c}
$$

Here small $m$ determine masses in solar units, $n_{51}=n_{f} / 10^{-51} \mathrm{~cm}^{-3}$. The approximate relation for dwarf mass - radius relation is used, which is valid for the ideal fermi gas stars, $R=7.6$. $10^{8} m_{d}^{-1 / 3}$. Everywhere carbon dwarfs with $\mu_{e}=2$ are considered, $\mu_{e}$ is the number of baryons per one electron. Following the evolution of the pair due to encounters it was found in [12] that in order to destroy the binary due to EE process the field star density should exceed

$$
n>3 \cdot 10^{5} \frac{m^{9 / 11}}{m_{f}^{2}} \mathrm{pc}^{-3} .
$$

The central parts of the most dense GC, where single recycled pulsars are concentrated, like M15, satisfy this demand. So, EE process is probably responsible for the formation of the single RP in $\mathrm{GC}$, as well as in the bulge, where they could find themselves after the evaporation of the whole GC. Note, that binaries with the hydrogen-helium brown dwarfs are destroyed easier due to EE [12], with the coefficient 2 instead of 3 in (4.6).

\section{Magnetic fields of NS in X-ray binaries}

There are several ways to estimate observationally magnetic field of an X-ray pulsar. During accretion matter is stopped by the magnetic field at the alfvenic surface, where gaseous and magnetic pressures are in a balance. At stationary state keplerian angular velocity of the accretion disk at the alfvenic surface is equal to the stellar angular velocity [75] $\Omega_{K}=\Omega_{s}=\Omega_{A}$. Otherwise neutron star would be accelerated due to absorption of matter with large angular momentum, or decelerated due to throwing away matter with additional angular momentum [43]. X-ray pulsars may have spin-up and spin-down stages [6], but most of them show average spin-up, what indicate to their angular velocity being less than the equilibrium one. This is observed in the best studied $\mathrm{X}$-ray pulsar Hex X-1 $[82,33]$. Analysis of spin-up/down phenomena in the X-ray pulsars indicate to important role of the mass loss [52], and to stochastic origin of spin-up/down transitions [53].

For a given luminosity $L_{36}=L /\left(10^{36} \mathrm{ergs} / \mathrm{s}\right)$ and dipole magnetic field at stellar equator $B_{12}=$ $B /\left(10^{12} \mathrm{G}\right)$ we get the following value of the equilibrium period [50], for the neutron star mass $M_{s}=1.4 M_{\odot}$, when a stellar rotational velocity is equal to keplerian rotational velocity at Alfven radius $r_{A}$ from (3.2)

$$
P_{e q} \approx 2.6 B_{12}^{6 / 7} L_{36}^{-3 / 7} \mathrm{~s} .
$$

This formula was derived for a disk accretion model, independently on a description of the viscosity. For Her X-1 parameters $L_{36}=10, P=1.24 \mathrm{~s}$, we get a magnetic field corresponding to the 
equilibrium rotation $B_{12}^{e q} \approx 1$. Taking into account the average spin-up of the pulsar in Her X-1, we may consider this value as an upper limit of its magnetic field. Even more rough estimations of the magnetic field in X-ray pulsars follow from the average spin-up rate under condition $\dot{J}_{r o t}=\dot{M} \Omega_{A}$, or restrictions on the polar magnetic field value following from the observed beam structure and condition of local luminosity not exceeding the critical Eddington one. These conditions lead to smaller values of the magnetic field of Her X-1 on the level $10^{9}-10^{10} \mathrm{G}[20,21,8]$.

Observations of low mass X-ray binaries (LMXB) indicate to very low values of their magnetic fields. Modulation of X-ray flux permitted to reveal the rotational period of the neutron star in the LMXB SAX J1808.4-3658 corresponding to the frequency $401 \mathrm{~Hz}$, due to RXTE observations (see reviews [91, 92]). This observations fill a gap and form a long-waiting link between LBXB and recycled millisecond pulsars [78], as neutron stars with very low magnetic field (up to $10^{8} \mathrm{G}$ ).

The most reliable estimation of the magnetic field of the X-pulsar Her X-1 comes from detailed observations of the beam variation in this pulsar on different stages, made on the satellites ASTRON [82], and GINGA [33] and RXTE [80]. This pulsar, in addition to $1.24 \mathrm{~s}$ period of the neutron star rotation, is in a binary system with an orbital period 1.7 days, and shows a 35 day cycle, where during only 12 days its luminosity is high. During other 23 days its X-ray luminosity strongly decreases, but small changes in the optical luminosity and strong reflection effect indicate, that the X-ray luminosity remains almost the same during all 35 day cycle. Visible decrease of the X-ray flux is due to an occultation phenomena. The model which explains satisfactory the phenomenon of the 35 day cycle is based on the precession of the accretion disc with the 35 day period, and occultation of the X-ray beam during 23 days. Analysis of the beam structure during high and low X-ray states lead to the conclusion, that during the low state we observe not the direct Xray flux from the neutron star, but the flux, reflected from the inner edge of the accretion disc. This conclusion is based on the $180 \mathrm{deg}$ phase shift between the $\mathrm{X}$-ray beams in high and low states $[82,33,80]$. In order to observe the X-ray flux reflected from the inner edge, situated near the Alfven radius of the accretion disc, it cannot be very far away from the neutron star. The estimations give the upper limit to the ratio of the Alfven and stellar radiuses

$$
\frac{r_{A}}{r_{s}} \leq 20
$$

The schematic picture of the accretion disc and its inner edge orientation around the neutron star at different stages of the 35 day cycle is shown in Fig.4, taken from [82]. As was indicated above, the value of the Alfven radius is determined by the neutron star mass $\left(M_{s}=1.4 M_{S}\right)$, mass flux $\dot{M}=3 \times 10^{16} \mathrm{~g} / \mathrm{s}$, corresponding to the luminosity $L=10^{37} \mathrm{ergs} / \mathrm{s}$, and the value of the magnetic field. Taking dipole radial dependence of the magnetic field $B=B_{s}\left(r_{s} / r\right)^{3}$, and neutron star radius $r_{s}=10^{6} \mathrm{~cm}$, we obtain the ratio in the form $r_{A} / r_{s} \approx 300 B_{12}^{4 / 7}$. To have this ratio not exceeding 20 we get an inequality $B \leq 3 \times 10^{10} \mathrm{G}$.

It was found in [88] a feature in the spectrum of Her X-1 at energies between 50 and $60 \mathrm{keV}$. Interpreting it as a cyclotron line feature according to $E_{X}=\frac{\hbar e B}{m_{e} c}$ leads to the value of the magnetic field $B_{c y c l}=(5-6) \times 10^{12} \mathrm{G}$, what is much higher than any other above mentioned estimations. Spectral features had been observed also in other X-ray sources. Recent observations on RXTE [40], and Beppo-SAX [79] of the pulsating transient 4U 0115+63 had shown a presence of 3 and 4 cyclotron harmonics features, corresponding to the magnetic field strength of $1.3 \times 10^{12} \mathrm{G}$. A 


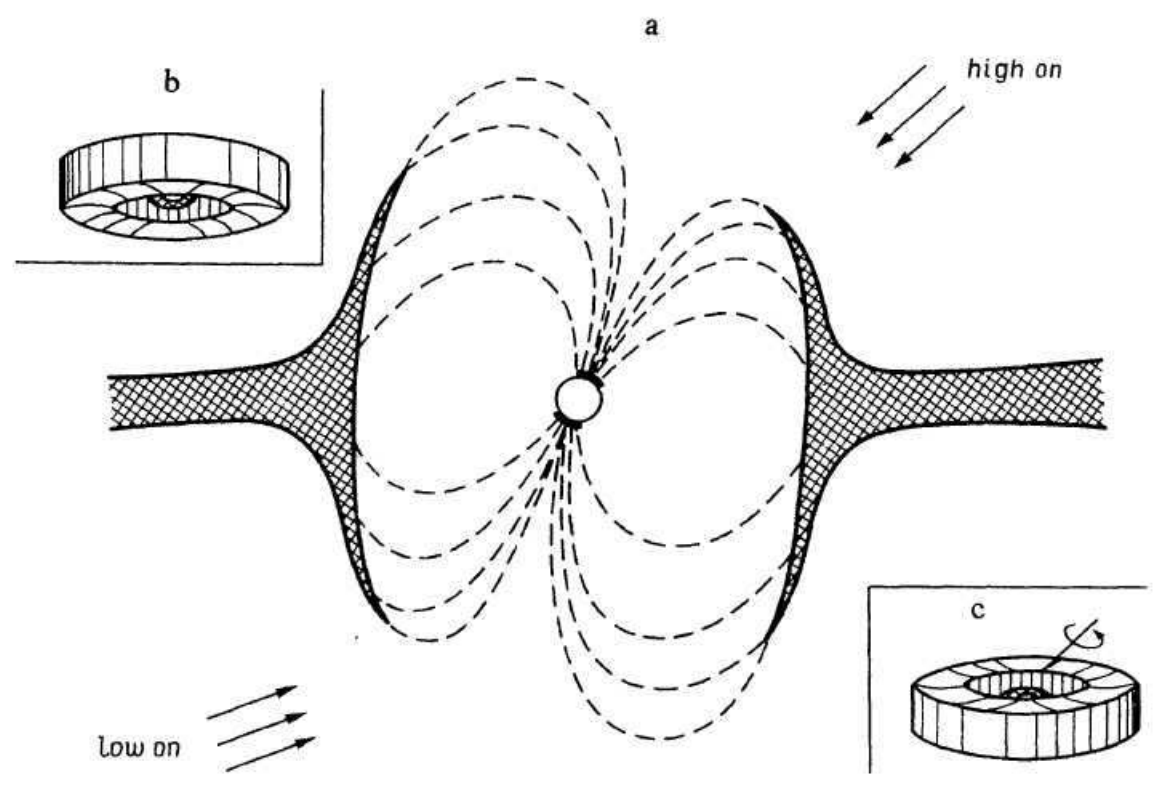

Figure 4: Configuration of the inner edge of the disk and the neutron star; neutron star and the disk in the "high-on" state (left top box), and in the "low-on" state (right bottom box), from [82].

comparison of the shapes of the beam in cyclotron harmonics may be used for testing the nature of these features. Cyclotron features had been observed in several X-ray sources [71], and they always had corresponded to large values of magnetic fields $B_{c y c l}>10^{12} \mathrm{G}$. Such situation was not in good accordance with a well established observational fact, that all recycled pulsars, going through a stage of an X-ray source, have much smaller magnetic fields, usually not exceeding $\sim 10^{10} \mathrm{G}$. Particularly, for the Her X-1 the value of its magnetic field, following from the cyclotron interpretation of the spectral feature, was in contradiction with all other observations, including the most reliable, based on the beam shape variability during 35 day cycle.

\section{Relativistic dipole interpretation of the spectral feature in Her X-1}

It seems likely that this conflict is created by using the non-relativistic formula connecting cyclotron frequency with a value of the magnetic field. According to [19], ultra-relativistic electrons, with a temperature $\sim 10^{11} \mathrm{~K}$, may be formed in the non-collisional shock during accretion, emitting a relativistic dipole line. The mean energy of this line is broadened and shifted relativistically, in comparison with the cyclotron line, by a factor of $2 \gamma \simeq \frac{2 k T}{m c^{2}}$. The spectrum profile of the relativistic dipole line is calculated in [3] for various electron distributions, where the model of the hot spot of Her X-1 is considered, and it is shown that the overall observed X-ray spectrum (from 0.2 to $120 \mathrm{KeV}$ ) can arise under the fields near $5 \cdot 10^{10} \mathrm{G}$ which are well below $B_{c y c l}$, and are not in the conflict with other observations.

According to [37, 7], in the magnetic field near the pulsar the cross component of a momentum of electrons is emitting rapidly, while the parallel velocity remains constant. Hence the momentum 
distribution of the electrons is anisotropic $p_{\perp}^{2} \ll p_{\|}^{2}$, with $p_{\perp} \ll m c, p_{\|} \gg m c$.
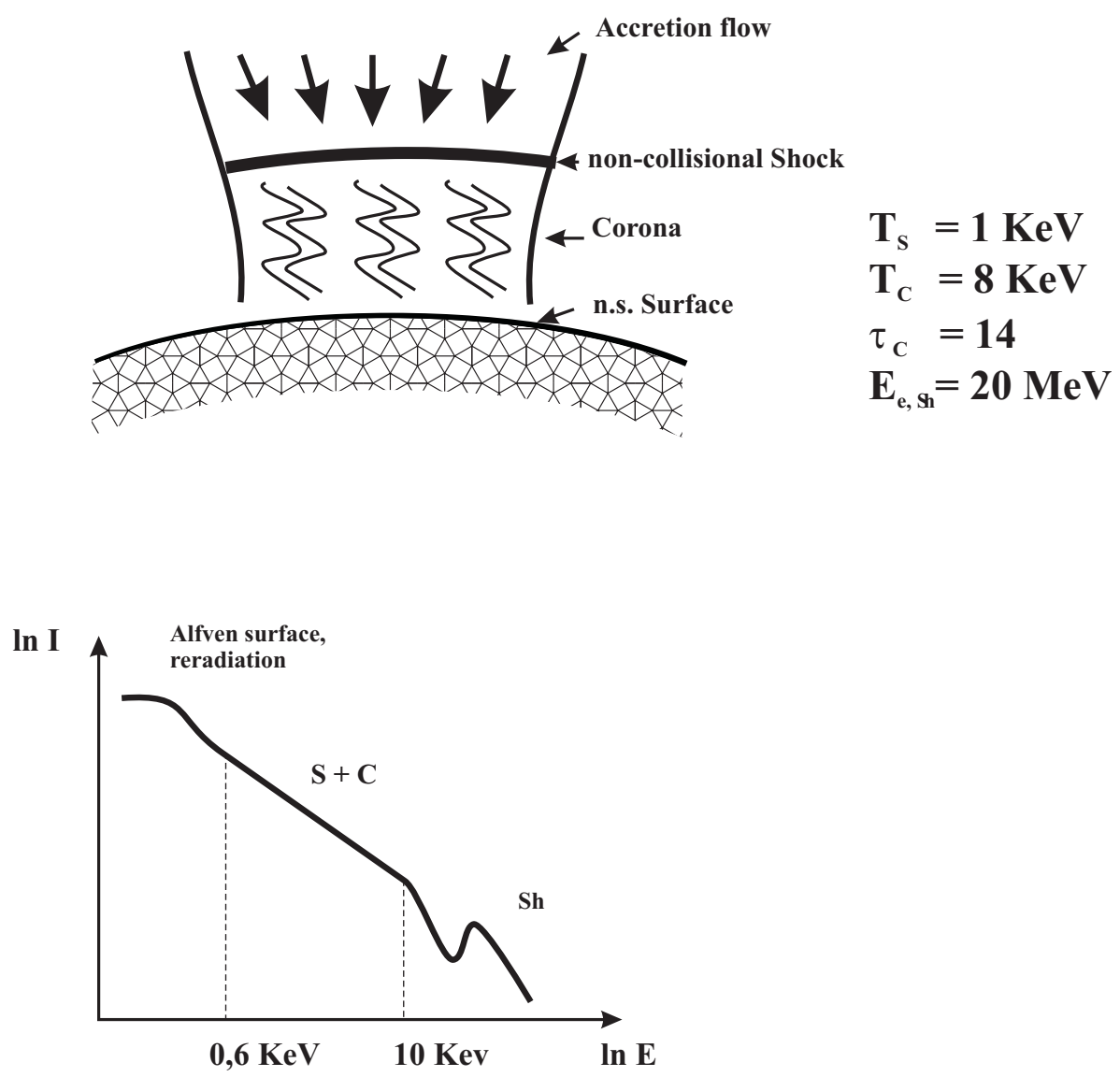

Figure 5: Schematic structure of the accretion column near the magnetic pole of the neutron star (top), and its radiation spectrum (bottom), from [15].

In order to obtain the whole experimental spectrum of the Her X-1 the following model of the hot spot (Fig.5) was considered in [3]. A collision-less shock wave is generated in the accretion flow near the surface on the magnetic pole of a neutron star. In it's front the ultra-relativistic electrons are generated. Under the shock there is a hot turbulent zone with a temperature $T_{e}$, and optical depth $\tau_{e}$, situated over a heated spot with a smaller temperature on the surface of the neutron star. The whole X-ray spectrum of pulsar Her X-1 from [66] is represented in Fig.6 by the solid line. There are three main regions in it: a quasi-Planckian spectrum between 0,3 and $0,6 \mathrm{KeV}$, that is generated (re-radiated) near the magnetosphere of the X-ray pulsar; power-law spectrum $(0.6 \div 20) \mathrm{KeV}$ with a rapid decrease at $20 \mathrm{KeV}$, and the "cyclotron" feature. The power-law spectrum is a result of comptonization in the corona of a black-body spectrum emitted by the stellar surface.

The comptonized spectrum has been calculated according to [85]. Setting the neutron star radius equal to $10 \mathrm{~km}$, distance to the X-ray pulsar $6 \mathrm{kps}$, hot spot area $S=2 \cdot 10^{12} \mathrm{~cm}^{2}$, the best fit was found at $T_{s}=1 \mathrm{keV}, T_{c}=8 \mathrm{keV}, \tau_{e}=14$, which is represented in Fig. 6 by the dashed line.

The experimental spectra taken from $[70,66]$ was approximated. The last spectrum (solid line) and its fitting (dashed line) are shown in Fig.6. The best fit for the line shape was obtained in [3] at the magnetic field strength $B=4 \cdot 10^{10} \mathrm{G}$. In this model the beam of the "cyclotron" feature is 


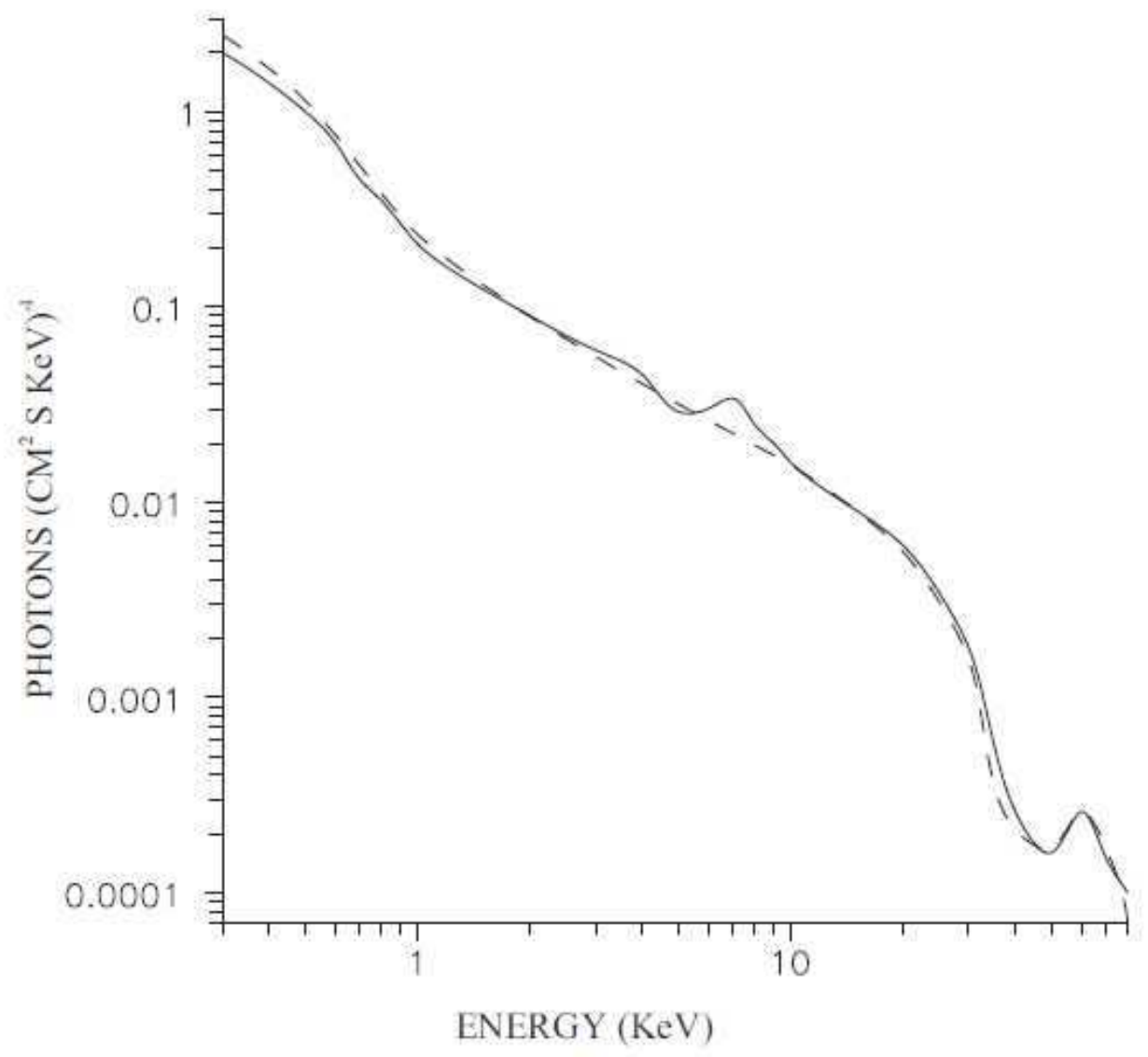

Figure 6: Comparison of the observational and computational X-ray spectra of Her X-1. The solid curve is the observational results taken from [66], the dot curve is the approximation with $T_{s}=0.9 \mathrm{KeV}, T_{e}=8 \mathrm{KeV}$, $\tau_{e}=14, B=4 \times 10^{10} \mathrm{G}$, from [3]

determined by the number distribution of the emitting relativistic electrons, moving predominantly along the magnetic field, over the polar cap.

The observations of the variability of the "cyclotron" lines are reported by [71]. Ginga detected the changes of the cyclotron energies in 4 pulsars. The change is as much as $40 \%$ in the case of $4 \mathrm{U}$ $0115+63$. Larger luminosity of the source corresponds to smaller average energy of the cyclotron feature. These changes might be easily explained in our model. The velocity of the accretion flow decreases with increasing of the pulsar luminosity because locally the luminosity is close to the Eddington limit. As a result the shock wave intensity drops as well as the energy of the ultrarelativistic electrons in it's front, leading to decrease of the relativistic dipole line energy.

\subsection{Polarization properties of magneto-dipole $\mathrm{X}$-ray lines}

The polarization properties of relativistic magneto-dipole radiation (RDR) in comparison with the cyclotron one are considered in [25], following [34]. For the cyclotron radiation the angular distribution of the radiation power and polarization level are given in Figs.7,8. The radiation of the relativistic electron with a factor $\gamma$, moving in the direction of the angle zero, is anisotropic, and concentrated inside the angle $\theta \sim 1 / \gamma$ around the velocity vector. When the angular resolution of 
the X-ray detector is worse than $1 / \gamma$, the whole relativistic magneto-dipole radiation is detected during the time when the beam is directed to the observer. This beam contains simultaneously the circular and linearly polarised photons, contrary to the cyclotron radiation, which produces a purely circularly polarised beam. the beam radiation is not monochromatic, with the spectral distribution shown in Fig.9.
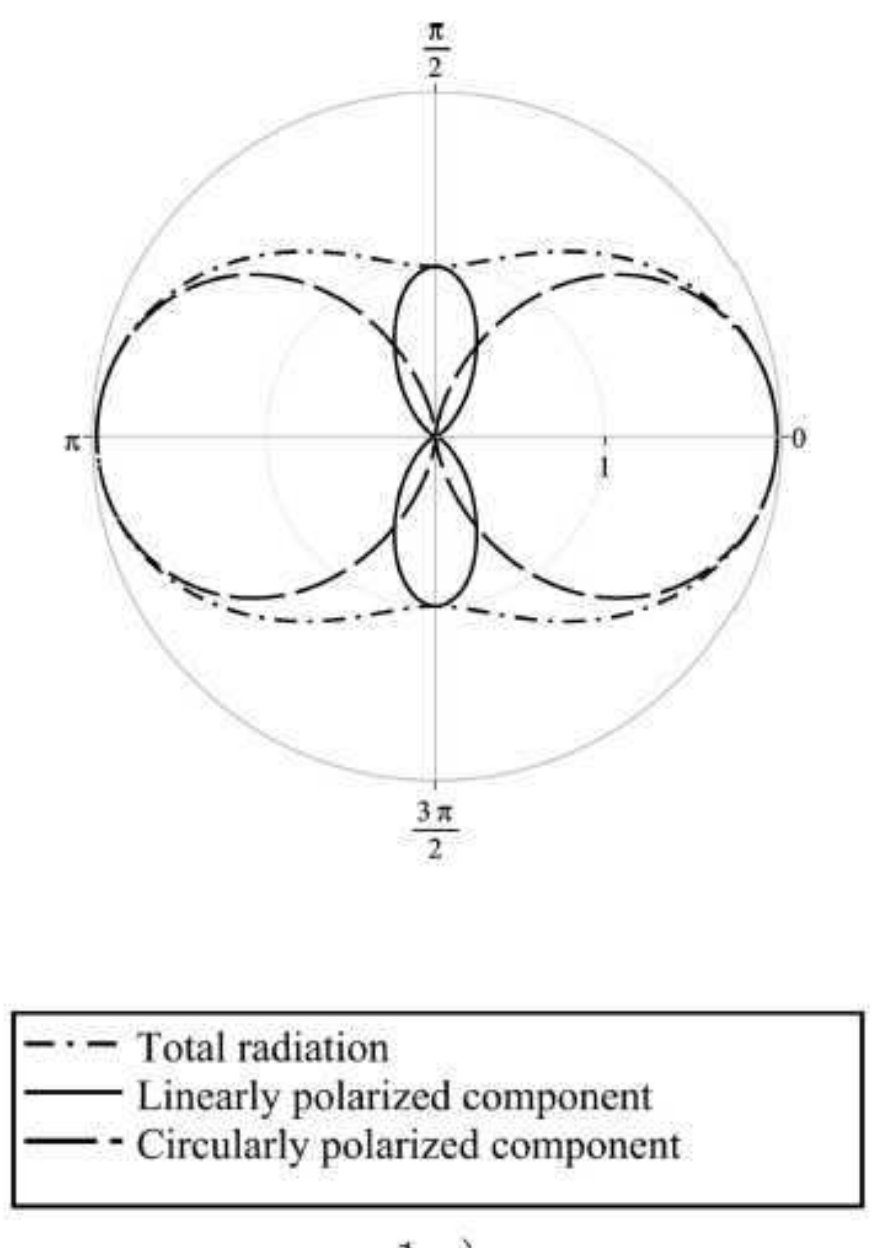

Figure 7: Angular distribution of cyclotron radiation polarization components, arbitrary units are used, from [25].

Measurements of polarization of the X ray feature in Her X1 would clarify its nature. Electrons with narrow momentum distribution across the magnetic field, will have in the direction of the magnetic pole, $\theta=0$, the following polarization properties [25]. The linearly polarized part of the radiation in the beam is equal to $R D R_{l} \approx 0.356$. The circularly polarized part of the beam radiation in the same direction, $\theta=0$, has different sign in the wavelength intervals $\frac{\omega_{B}}{2 \gamma_{\|}}<\omega<\gamma_{\|} \omega_{B}(0<x<$ $1)$, and $\omega_{B} \gamma_{\|}<\omega<2 \gamma_{\|} \omega_{B}(1<x<2), x=\frac{\omega}{\gamma_{\|} \omega_{B}}$. The circularly polarized part of the radiation at lower frequencies is equal to $R D R_{c 1} \approx 0.418(0<x<1)$, and at higher frequency $R D R_{c 2} \approx 0.707$ $(1<x<2)$, with opposite sign of polarisation. The polarization of the monochromatic cyclotron line in the direction $\theta=0$ is $100 \%$ circular, see Fig.7. In the RDR model the radiation is has a 


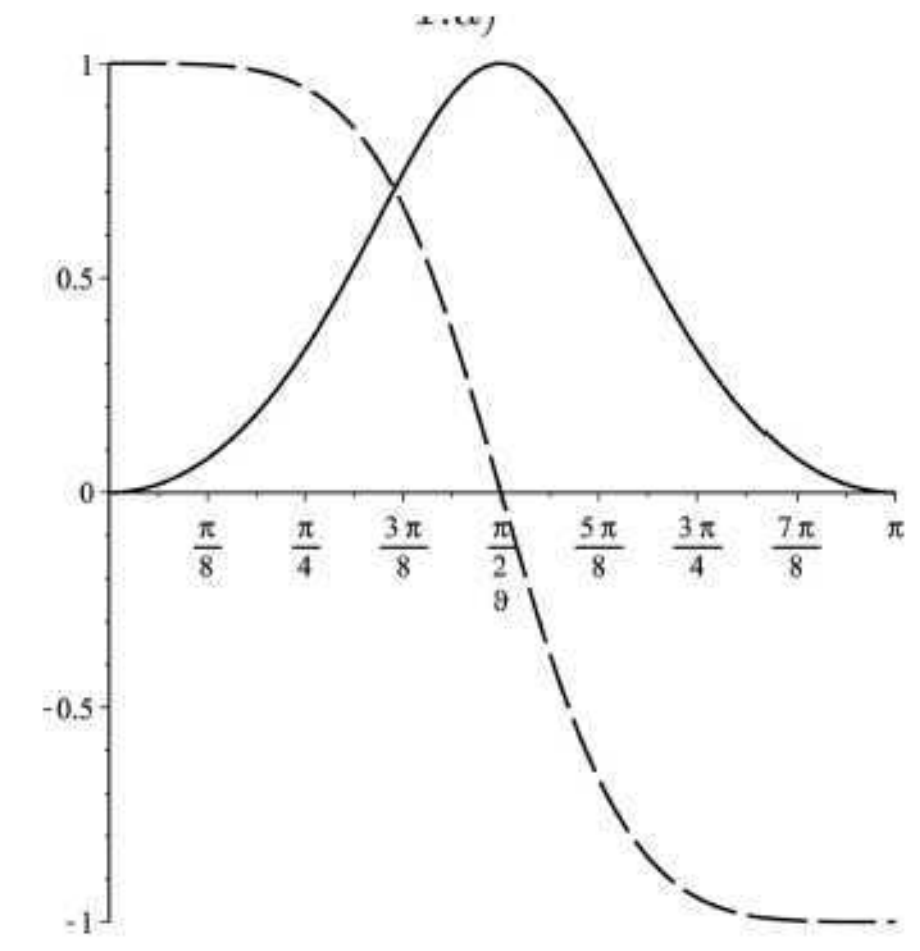

\section{- Linear polarization degree - - Circular polarization degree}

Figure 8: Angular dependence of the linear and circular polarization degrees of cyclotron radiation, arbitrary units are used, from [25].

spectrum, shown in Fig.9 with maximum at $\omega=2 \gamma \omega_{B}$. It may correspond to the width of the observed feature [88].

\section{Magnetic fields of soft gamma repeaters}

Soft gamma repeaters (SGR) are slowly rotating neutron star, with periods $P=2-8 \mathrm{~s}$. They are characterised by violent behaviour, with X-ray and $\gamma$-ray outbursts. The number of SGR is about 10 objects, and in 4 of them short giant bursts have been observed with harder spectrum, and $10^{4}-10^{6}$ times larger luminosity. The periods of SGR increase with time, but the rate of the loss of rotational energy is much less than the average luminosity of these objects. The additional source of energy in the form of magnetic reconnection was suggested [31, 32], in which the strength of the magnetic field should be $10^{14}-10^{15} \mathrm{G}$, what is much higher than the average magnetic field of radio pulsars. These objects have been called as magnetars.

The pulse shape is changing from one epoch to another, inducing errors in finding derivative of the period. The big jump in $\dot{P}$, visible in Fig 12 looks out surprising. for magnetic dipole losses, 


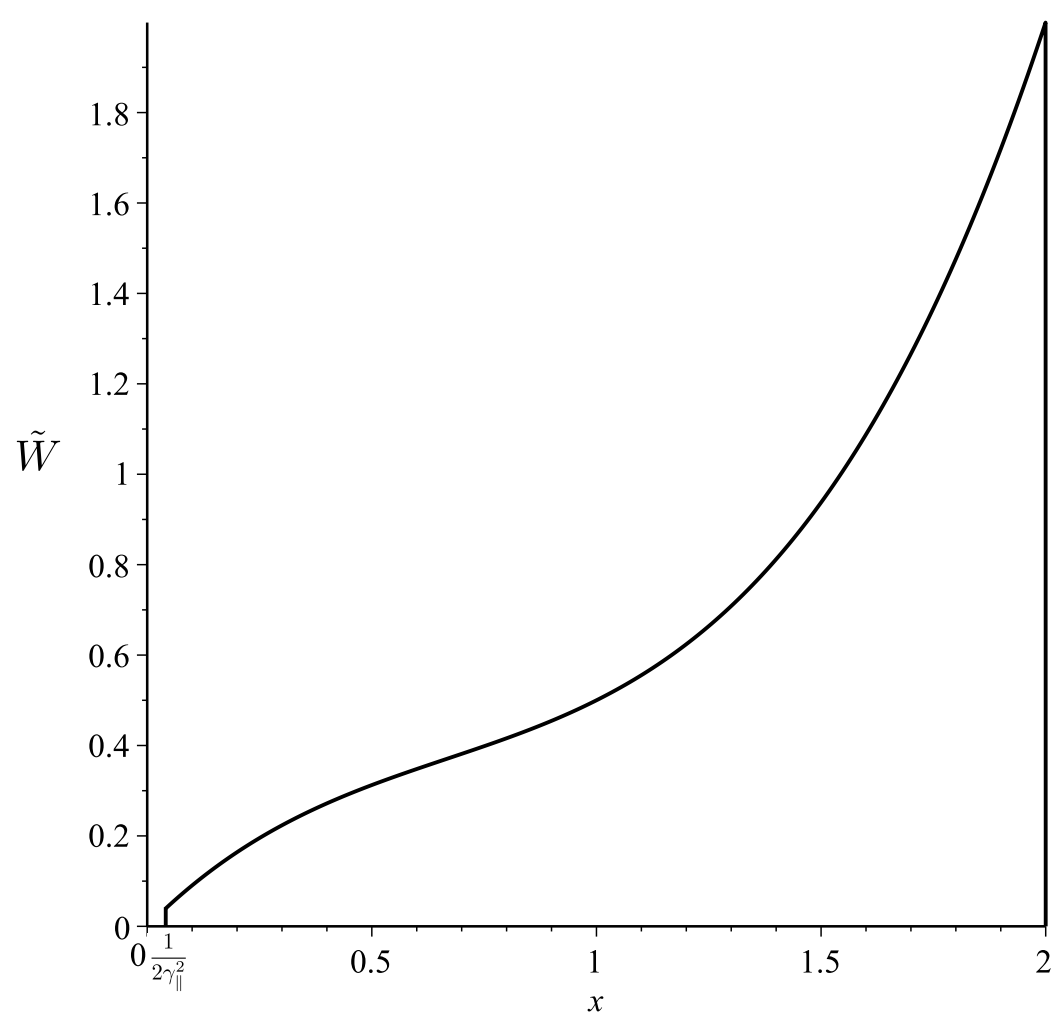

Figure 9: The frequency distribution of energy in the RDR line, from [25]

because it needs a considerable jump in the magnetic field strength, prohibited by self induction effects. Contrary, in the model of a pulsar wind rotational energy losses it looks quite reasonable, that these losses strongly increase during the giant burst, when the $\dot{P}$ jump was observed [16].

\subsection{Giant bursts}

Giant bursts had been observed in four SGR sources, when the peak luminosity increased 4 âĂŞ 6 orders of magnitude. In presence of slow rotation, and low rotational energy, even average observed luminosity exceeds rotational loss of energy more than 10 times, and orders of magnitude during giant outbursts. The energies emitted in the giant bursts, in the four SGR, during a time not exceeding few hundreds of seconds, have values

1. The giant burst from the source SGR 0526-66 in Large Magelanic Cloud was discovered by Mazets et al. at 5 March 1979, with total energy exceeding $2 \cdot 10^{44} \mathrm{erg}[59,60]$.

2. SGR 1900+14 was discovered by Mazets et al. in 1979 [58], (Giant burst happened at 27 August 1998, with total energy exceeding $\left.7 \cdot 10^{43} \mathrm{erg}[64,65]\right)$.

3. SGR 1806-20 was observed by Mazets et al. in 1980 [61, 62], and was identified as SGR in [49] (Giant burst happened at 27 December 2006, with total energy

$\left.\sim 2 \cdot 10^{46} \operatorname{erg}[61,62]\right)$.

4. Semi-giant burst SGR 1627-41 was discovered by BATSE at 18 June 1998, with total energy $\sim 10^{43} \mathrm{erg}[46,63]$. 


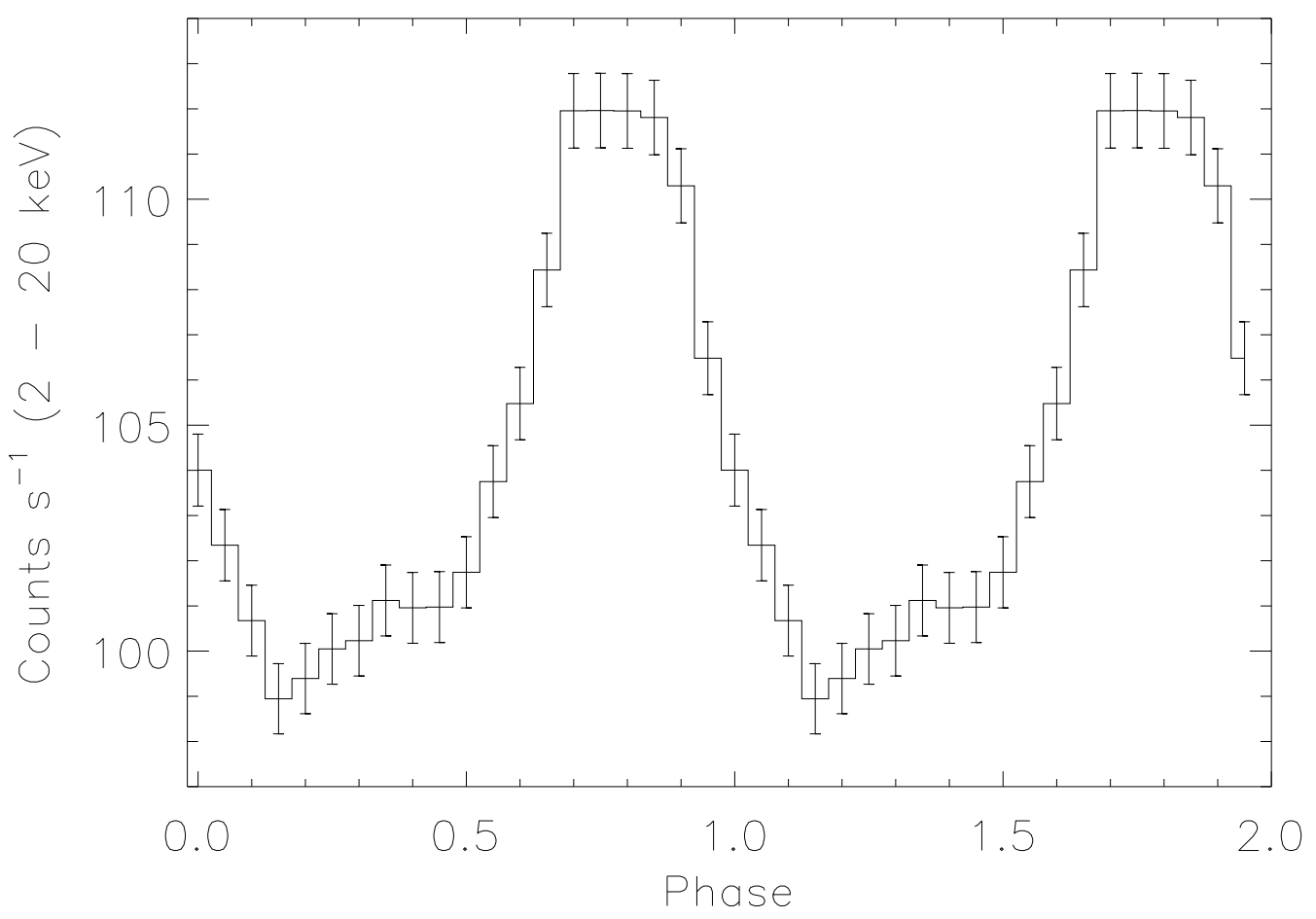

Figure 10: The epoch folded pulse profile of SGR $1900+14(2-20 \mathrm{keV})$ for the May $1998 \mathrm{RXTE}$ observations, from [47].

\subsection{Estimations of the magnetic fields in SGR}

Despite the fact, that rotation energy losses are much smaller than the observed luminosity, for an estimation of the magnetic field strength in these objects the same procedure was used, as in radio pulsars, based on measurements of $P$ and $\dot{P}$, and using (2.1). The measurements have been done for SGR $1900+14$, in different epochs, using data from the satellites RXTE and ASCA [47], which are presented in Figs.10-12. The drastic change in the pulse shape is leading to big errors in the derivative of the period, so the magnetic field in this object was estimated as $(2 \div 8) \cdot 10^{14} \mathrm{G}$.

The radio pulsars, where the value of the magnetic field is measured more precisely, may rotate slowly, have very large magnetic fields, but show no difference in their behaviour in comparison with other radio pulsars. These are radio pulsars with measured "magnetar" fields, up to $10^{14} \mathrm{G}$ : PSRs J1847-0130 and J1780-37184 [67, 68].

From another side, the source SGR 0418+5729 was detected in the X-ray observations, and it was concluded that its dipolar magnetic field cannot be greater than $7.510^{12}$ Gauss, well in the range of ordinary radio pulsars, implying that a high surface dipolar magnetic field is not necessarily required for magnetar-like activity in [77].

Rotation energy losses are much less than observed luminosity in SGRs, so estimations of the magnetic field strength, using highly variable values of $\dot{P}$ is not a correct procedure. Jumps in $\dot{P}$, in the pulse form are not seen in in any radio pulsars. But increase of $\dot{P}$, due to increase of magnetic 


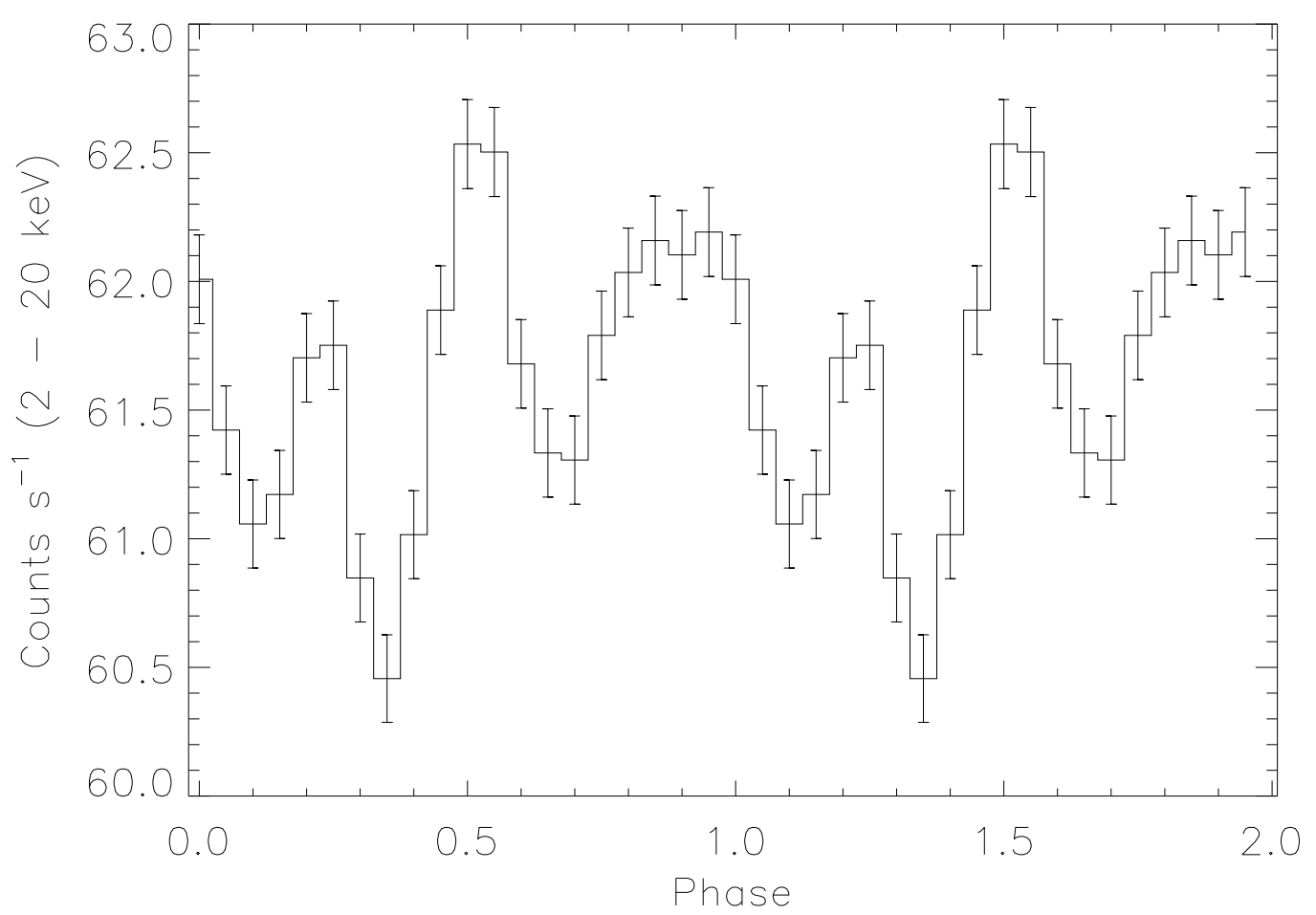

Figure 11: The epoch folded pulse profile of SGR $1900+14(2-20 \mathrm{keV})$ for the August 28, 1998 RXTE observation. The plot is exhibiting two phase cycles, from [47].

wind during giant burst, is expected, so rotation energy losses by magnetic wind explain the period increase, and the values of neutron star magnetic fields is SGR are similar to those of ordinary radio pulsars [16].

\section{Nuclear explosions near neuron star surface}

Analysis of a neutron star (NS) formation during collapse had shown, that cooling of the neutron star does not lead to thermodynamic equilibrium in the outer NS crust, where a layer with non-equilibrium composition is formed [17]. The energy stored in this layer may be released when its matter is moved to NS surface as a result of starquake [18], or development of another kind of instability [24]. Such explosions, connected with development of fission reaction of superheavy nuclei from non-equilibrium layer may be observed as a special type of gamma ray bursts, connected with SGR $[18,24]$. The energy $E_{n}$, stored in this layer strongly depends on the NS mass. For characteristic mass $M_{N S}=1.4 M_{\odot}$ this energy is $E_{n} \sim 10^{48} \mathrm{erg}$ [17]. The mass of the nonequilibrium layer is larger for low-mass NS, because of lower gravity leading to larger thickness of this layer. It is suggested in [24], that SGR activity is connected with instabilities, developed in the low-mass NS crust with $M_{N S}<0.8 M_{o} d o t$, connected with magnetic fallback accretion of the 


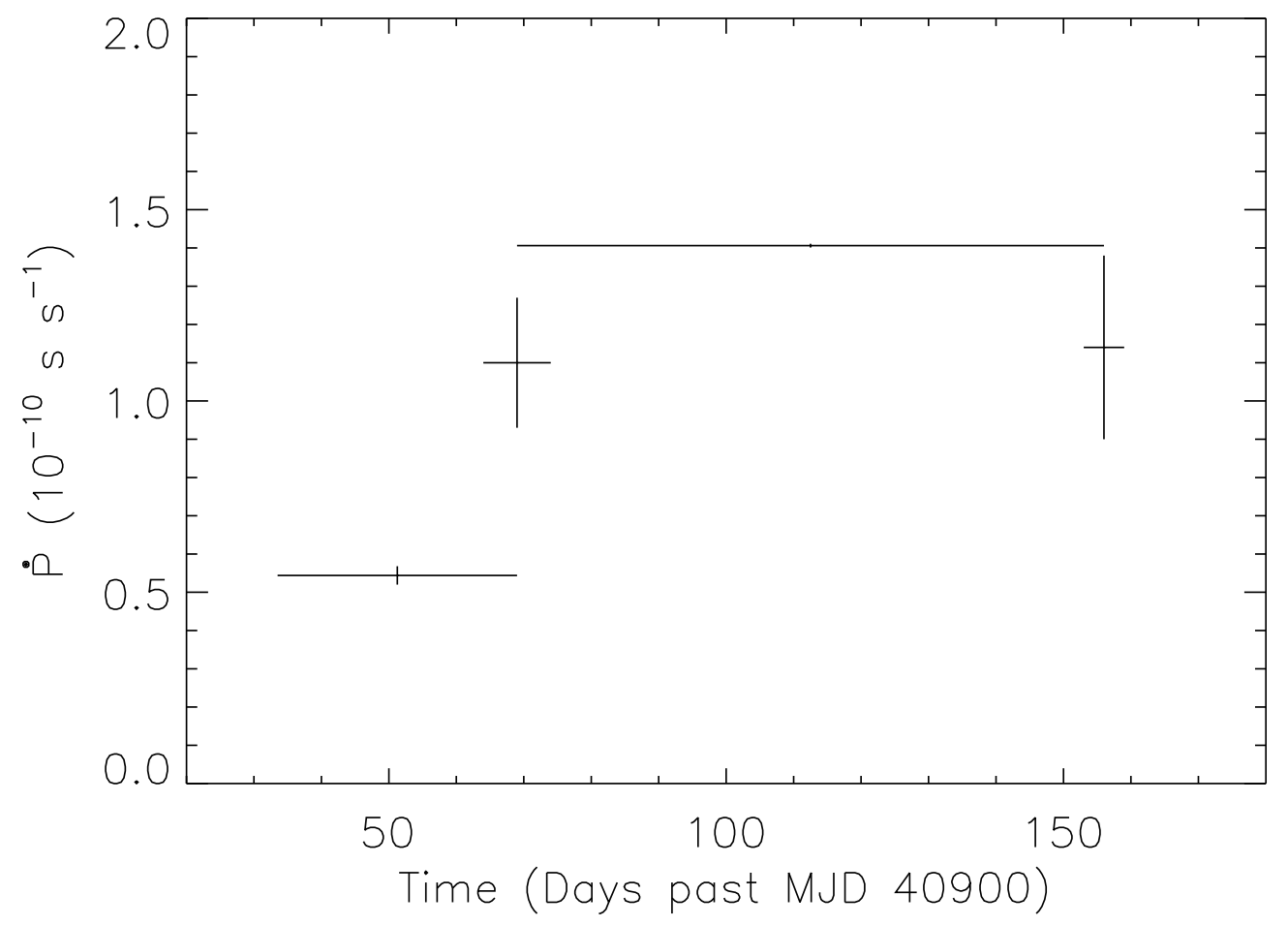

Figure 12: The evolution of "Period derivative" versus time since the first period measurement of SGR 1900+14 with ASCA in [42]. The time is given in Modified Julian Days (MJDs), from [47].

matter after SN explosion. According to estimations the energy store in the non-equilibrium layer is enough for producing $\sim 200$ giant bursts.

\section{Conclusions}

1. Magnetic fields of radio pulsars are in good correspondence with theoretical estimations. Magnetic fields of single radio pulsars are damping very slow, if any.

2. RP and LMXB have small magnetic fields, which very probably had been decreased by screening during the accretion stage.

3. Contradiction between high $B_{c y c l}$ and other observational estimations of $B$ in the LMXB Her X-1 may be removed in the model of relativistic dipole mechanism of the formation of a hard spectral feature by strongly anisotropic relativistic electrons, leading to conventional value of $B \approx 5 \cdot 10^{10} \mathrm{G}$.

4. Very high magnetic fields in magnetar model of SGR needs farther confirmation and investigation.

5. Non-equilibrium layer is formed in the neutron star crust, during NS cooling, or during accretion onto it. It may be important for NS cooling, glitches, and explosions connected with SGR. 


\section{Acknowledgement}

The work was partially supported by grants RFBR 17-02-00760, 18-02-00619; and Presidium RAS programm I.28P.

\section{References}

[1] Aarseth, S.J., Lecar, M., Ann. Rev. Astron. Ap. 13(1975) 1.

[2] Amnue'l, P.R., Guseinov, O.Kh., Astron. Tsirk. No.524 (1969).

[3] Baushev, A.N., Bisnovatyi-Kogan, G.S., Astron. Reports 43 (1999) 241.

[4] Bhattacharya, D., van den Heuvel, E.P.J. "Formation and evolution of binary and millisecond radio pulsars." Physics Reports 203 (1991) 1-124.

[5] Bhattacharya, D., Wijers, R A.M.J., Hartman, J.W., Verbunt, F., Astron. Ap., 254 (1992) 198.

[6] Bildsten, L., Chakrabarty, D., Chiu, J., et al., ApJ Suppl. 113 (1997) 367.

[7] Bisnovatyi-Kogan, G.S., Astron. Zh. 50 (1973) 902.

[8] Bisnovatyi-Kogan, G.S., Astron. Zh. 51 (1974) 443.

[9] Bisnovatyi-Kogan, G.S., Priroda (Nature), No.3 (March 1975), p.100 (in Russian).

[10] Bisnovatyi-Kogan, G.S. "Magnetohydrodynamical processes near compact objects" Nuovo Cimento, Rivista, Serie 3, vol. 2, no. 1 (1979) 1-37.

[11] Bisnovatyi-Kogan, G.S., Astrophysics 31 (1990) 751; 32 (1990) 103.

[12] Bisnovatyi-Kogan, G.S., Astrophysics 32 (1990) 176.

[13] Bisnovatyi-Kogan, G.S., Mem. Soc. Astron. Ital. 73 (2002) 318; astro-ph/9911275.

[14] Bisnovatyi-Kogan, G.S. Binary and recycled pulsars: 30 years after observational discovery. Physics Uspekhi 49 (2006) 53-67.

[15] Bisnovatyi-Kogan, G.S. Evolutions of Neutron Stars and their Magnetic Fields. in Neutron Stars, Supernovae and Supernova Remnants, p.109. Eds. by O.H. Guseinov, E. Yazgan and A. Ankay,(Nova Science Publishers, New York, 2007).

[16] Bisnovatyi-Kogan, G.S. Young neutron stars with soft gamma ray emission and anomalous X-ray pulsar. in Handbook of Supernovae, p.1401. Eds. A.W. Alsabti, P. Murdin, (Springer, 2017).

[17] Bisnovatyi-Kogan, G.S., Chechetkin, V.M., Astrophys. Space Sci. 26 (1974) 25.

[18] Bisnovatyi-Kogan, G.S., Imshennik, V. S., Nadyozhin, D. K., Chechetkin, V.M., Astrophys. Space Sci. 35 (1975) 23.

[19] Bisnovatyi-Kogan, G.S., Fridman, A.M., Astron. Zh. 46 (1969) 721.

[20] Bisnovatyi-Kogan, G.S., Komberg, B.V., Astron. Zh. 51 (1974) 373.

[21] Bisnovatyi-Kogan, G.S., Komberg, B.V., Astron. Zh. 52 (1975) 457.

[22] Bisnovatyi-Kogan, G.S., Komberg, B.V., Astron. Zh. Pisma 2 (1976) 338.

[23] Bisnovatyi-Kogan, G.S., Romanova, M.M., Sov. Astron. 27 (1983) 519.

[24] Bisnovatyi-Kogan, G.S., Ikhsanov, N.R., Astronomy Reports 58 (2014) 217. 
[25] Bisnovatyi-Kogan, G.S. and Lyakhova, Ys.S., MNRAS 456 (2016) 3186.

[26] Burgay, M., D’Amico, N., Possenti, A., et al., Nature 426 (2003) 531.

[27] Cheng, K.S., Zhang, C.M., Astron. Astrophys. 337 (1998) 441.

[28] Cheng, K.S., Zhang, C.M., Astron. Astrophys. 361 (2000) 1001

[29] Choudhuri, A.R., Konar, S., Mon.Not.Roy.Astron.Soc. 332 (2002) 933.

[30] Choudhuri, A.R., Konar, S., Current Science 86 (2004) 444.

[31] Duncan, R.C., Thompson, C., ApJ Lett. 392 (1992) L9.

[32] Duncan, R.C., Thompson, C., MNRAS 275 (1995) 255.

[33] Deeter, J.E., Scott, D.M., Boynton, P.E., et al., ApJ 502 (1998) 802.

[34] Epstein, R., ApJ 183 (1973) 593.

[35] Fruchter, A.S., Stinebring, D.R., Taylor, J.H., Nature 333 (1988) 237.

[36] Ginzburg, V.L., Dokl. Akad. Sci. USSR 156 (1964) 43.

[37] Gnedin, Yu.N., Sunyaev, R.A., Astron. Ap. 25 (1973) 233.

[38] Goldreich, P., Julian, W., ApJ 157 (1969) 869.

[39] Gurevich, L.E., Levin, B.Yu., Asnron. Zh. 27 (1950) 273.

[40] Heindl, W.A., Coburn, W., Gruber, D.E., et al., Astrophys. J. Lett. 521 (1999) L49.

[41] Hulse, R.A., Taylor, J.H., Astrophys. J. Lett. 195 ( 1975) L51.

[42] Hurley, K., Cline, T., Mazets, E., Barthelmy, S., Nature 397 (1999) 41.

[43] Illarionov, A.F., Sunyaev, R.A., Astron. Ap. 39 (1975) 185.

[44] Kluzniak, W., Ruderman, M., Shaham, J., Tavani, M., Nature 334 (1988) 225.

[45] Konar, S., Choudhuri, A.R., Mon. Not. R.A.S. 348 (2004) 661.

[46] Kouveliotou, C., Kippen, M., Woods, P., et al., The Astronomer's Telegram (1998) âĎÚ 29.

[47] Kouveliotou, C., Strohmayer, T., Hurley, K., et al., ApJ Lett. 510 (1999) L115.

[48] Landau, L.D., Lifshitz, E.M. Teoriya Polya. Nauka, Moscow (1962).

[49] Laros J.G., Fenimore E.E., Fikani M.M., et al., Nature 322 (1986) 152.

[50] Lipunov, V.M. Astrophysics of Neutron Stars. Springer, Germany (1992).

[51] Lorimer, D. R., "Binary and Millisecond Pulsars". Living Reviews in Relativity 11 (2008) 8.

[52] Lovelace, R.V.L, Romanova, M.M., Bisnovatyi-Kogan, G.S., Month. Not. R.A.S. 275 (1995) 244.

[53] Lovelace, R.V.L, Romanova, M.M., Bisnovatyi-Kogan, G.S., ApJ 514(1999) 368.

[54] Lovelace, R.V.L, Romanova, M.M., Bisnovatyi-Kogan, G.S., ApJ 625 (2005) 957.

[55] Lyne, A.G., Graham-Smith, F. Pulsar Astronomy. Cambridge Univ. Press, UK (1998).

[56] Lyne, A.G., Burgay, M., Kramer, M., et al., Science 303 (2004) 1153.

[57] Manchester, R.N., Taylor, J.H., Astronomical Journal 86 (1981) 1953.

[58] Mazets, E.P., Golenetskii, S.V., Gur’yan, Yu.A., Sov. Astron. Lett. 5 (1979) 343. 
[59] Mazets, E.P., Golenetskii, S.V., Il'Inskii, V.N., et al. Sov. Astron. Lett. 5 (1979) 163.

[60] Mazets, E.P., Golentskii, S.V., Ilinskii, V.N., et al., Nature 282 (1979) 587.

[61] Mazets, E.P., Golenetskii, S.V., Il'inskii, V.N., et al. Astrophys. Space Sci. 80 (1981) 3.

[62] Mazets, E.P., Golenetskii, S.V., Guryan Yu, A. \& Ilyinski, V.N., Astrophys. Space Sci. 84 (1982) 173.

[63] Mazets, E.P., Aptekar, R.L., Butterworth, P., et al., ApJ Lett 519 (1999) L151.

[64] Mazets, E.P., Cline, T.L., Aptekar, R.L., et al., Astron. Lett. 25 (1999) 628.

[65] Mazets, E.P., Cline, T.L., Aptekar, R.L., et al., Astron. Lett. 25 (1999) 635.

[66] McCray, R.A., Shull, J.M., Boynton, P.E., et al., ApJ 262 (1982) 301.

[67] McLaughlin, M.A., Stairs, I.H., Kaspi, V.M., et al., ApJ Lett. 59l (2003) L135.

[68] McLaughlin, M.A., Lorimer, D.R., Lyne, A G., et al.,in IAU Symposium no. 218, held as part of the IAU General Assembly, 14-17 July, 2003 in Sydney, Australia. Edited by Fernando Camilo and Bryan M. Gaensler. San Francisco, CA: Astronomical Society of the Pacific (2004) pp.255-256.

[69] Michel, F.C., Mon. Not. R.A.S. 267 (1994) L4.

[70] Mihara, T., Makishima, K., Ohashi, T., et al., Nature 346 (1990) 250.

[71] Mihara, T., Makishima, K., Nagase, F., Proc. Workshop "All-sky X-ray Observations in the next decade” (1997) p.135.

[72] Muslimov, A., Page, D., Astrophys. J. Lett. 440 (1995) L77.

[73] Pacini, F., Nature 216 (1967) 567.

[74] Paczynski, B., Acta Astronomica 31 (1981) 1.

[75] Pringle, J., Rees, M., Astron. Ap. 21 (1972) 1.

[76] Rappaport, S., Putney, A., Verbunt, F., Astrophys.J 345 (1989) 210.

[77] Rea, N., Esposito, P., Turolla, R., et al., Science 330 (2010) 944.

[78] Ruderman, M., Shaham, J., Comments on Astrophys. 10 ( 1983) 15.

[79] Santangelo A., Segreto A., Giarrusso S., et al., Astrophys. J. Lett. 523 (1999) L85.

[80] Scott, D.M., Leahy, D.A., Wilson, R.B., ApJ 539 (2000) 392.

[81] Shapiro, S. L., Teukolsky, S. A., Black Holes, White Dwarfs and Neutron Stars. John Wiley \& Sons ( 1983).

[82] Sheffer, E.K., Kopaeva, I.F., Averintsev, M.B., et.al., Sov. Astron. 36 (1992) 41.

[83] Shklovsky, I.S., Comments on Astrophys. 9 (1982) 261.

[84] Spitzer, L., Dynamical evolution of globular clusters Princeton University Press. (1987) 191 pp.

[85] Sunyaev, R.A., Titarchuk, L.G., Astron. Ap. 86 (1980) 121.

[86] Taylor, J H., Hulse, R.A., Fowler, L.A., et al., ApJ Lett. 206 (1976) L53.

[87] Trimble, V., Rees, M., The Crab Nebula. in Proceedings from IAU Symposium no. 46 held at Jodrell Bank, England, August 5-7. Eds. R. D. Davies and F. Graham-Smith. Dordrecht, Reidel (1970) p.273.

[88] Trümper, J., Pietsch, W., Reppin, C., et al., ApJ Lett. 219 (1978) L105. 
[89] Tutukov, A., Yungelson, L., Nauchnye Informatsii 27 (1973) 86.

[90] van den Heuvel, E.P.J., Bitzaraki, O., Astron. Astrophys. 297 (1995) L41.

[91] Van der Klis, M., astro-ph/9812395 (1998).

[92] Wijnands, R., astro-ph/0403409 (2004) .

[93] Zapolsky, H.S., Salpeter, E E., Astrophys. J. 158 (1969) 809. 